\title{
Genetic basis of sperm and testis length differences and epistatic effect on hybrid inviability and sperm motility between Drosophila simulans and D. sechellia
}

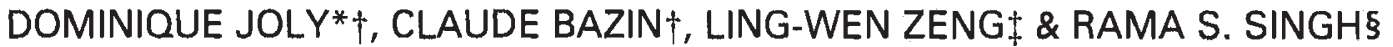 \\ $\nmid$ Laboratoire Populations, Génétique et Evolution, CNRS 91198 Gif sur Yvette Cedex, France, †Department of \\ Ecology and Evolution, 1101 E. 57th St., The University of Chicago, Chicago, 12 60637, USA and §Department of \\ Biology, MacMaster University, 1280 Main Street West, Hamilton, Ontario, Canada L8S $4 K 1$
}

\begin{abstract}
Results are reported from a genetic study of hybrid inviability and three 'fertilization traits' (sperm motility and length, and testis size) that affect hybrid sterility between the sibling species Drosophila simulans and $D$. sechellia. The main findings are as follows. (i) For sperm length there was a dominant effect of the $D$. simulans genome over that of $D$. sechellia, and the Y chromosome of $D$. sechellia in the background of $D$. simulans reduced the sperm length. (ii) In contrast, testis length, in spite of its generally high correlation with sperm length, showed an additive effect. (iii) We found a strong asymmetric incompatibility between the $D$. sechellia $\mathrm{X}$ chromosome and $D$. simulans autosomes: $D$. sechellia $\mathrm{X}$ chromosome with $D$. simulans autosomes, but not the reverse, showed a significant reduction in testis length as well as in hybrid inviability compared to the parental species. (iv) Between the two autosomes, chromosome 3 had a greater effect on these traits than chromosome 2 , and there was additionally an epistatic effect between these chromosomes with respect to their parental vs. recombinant status: recombinant chromosomes 2 and 3, together, had lower viability than any other combination. (v) The testis size in the backcross generation was greater than the parental species, suggesting that some modifier genes are being released from their species-specific genetic control. (vi) The species-specific homogeneity of the genome was important for all three traits-offspring viability, hybrid male fertility and testis length. These results are discussed with respect to the role of sexual selection and genetic divergence during speciation.
\end{abstract}

Keywords: Drosophila, epistatic effect, speciation, species differences, sperm length, testis length.

\section{Introduction}

The genetic mechanisms of species formation is one of the most challenging problems in evolutionary biology and we have seen the resurgence of a renewed interest in these mechanisms (see Orr, 1991; Coyne, 1992; Pantazidis et al., 1993). One way to approach this problem is to study the genetic basis of reproductive isolation between related species and this has currently become the favoured experimental approach (Dobzhansky, 1936; Zouros, 1981; Wu \& Beckenbach, 1983; Coyne, 1984, 1985, 1993; Coyne \& Kreitman, 1986; Orr, 1987, 1989;

*Correspondence. E-mail: joly@sunbge.bge.cnrs-gif.fr
Zouros et al., 1988; Zeng \& Singh, 1993a,b, 1995). Previous studies have generally focused on hybrid male sterility and relatively little attention has been paid to hybrid inviability; this approach was intended to identify genes affecting hybrid sterility. Species differ in more than reproductive isolation, so a broader approach, including all traits affecting reproduction, is required. This report combines the two approaches. Sperm length is an excellent trait for studying species differences as it is speciesspecific and is the most rapidly evolving trait in insects (Joly et al., 1989, 1991). Drosophila species are good candidates on which to perform such an investigation because they encompass the extreme range of sperm length variation in all invertebrates, 
ranging from $33 \mu \mathrm{m}$ in $D$. subobscura (Joly et al., 1989 ) to nearly $6 \mathrm{~cm}$ in $D$. bifurca (Joly et al., 1995).

This study reports the genetic analysis of hybrid inviability and three strongly related fertilization traits, sperm length and motility and testis size, in the sibling species $D$. simulans and $D$. sechellia from the melanogaster complex. These species are closely related (Coyne, 1984; Lachaise et al., 1986). Their chromosomes are entirely homosequential and crosses ( $D$. simulans female by $D$. sechellia males) can be easily made giving fertile, hybrid females and sterile, hybrid males (Lemeunier \& Ashburner, 1984). Drosophila sechellia sperm length has strongly diverged from that of the mauritiana-simulans pair, being almost one and a half times as long as that of D. simulans (1.6 and $1.2 \mathrm{~mm}$, respectively; Joly, 1987).

The results show interesting differences in the effects of sex chromosomes, autosomes and their interactions on hybrid inviability and sterility, sperm length and motility and testis size.

\section{Material and methods}

\section{Species stocks, genetic markers and crossing scheme}

A D. simulans strain homozygous for a recessive mutant marker on each of the five major chromosome arms, and a $D$. sechellia strain (Robertson) were supplied courtesy of Dr Jerry Coyne. The five mutant markers are forked -2 on the $\mathrm{X}$ chromosome $\left(f^{2}: \mathrm{I}-60\right)$, net and plum on the second chromosome (nt: II-2, 2L; pm: II-103, 2R), and scarlet and ebony on the third chromosome (st: III-44, 3L; $e$ : III-71, $3 R$ ). The tiny fourth chromosome was not studied because no marker was available. The crossing scheme is the same as used by Dobzhansky (1937) and others (Coyne, 1985; Coyne \& Kreitman, 1986; Orr, 1989). Females of the marker strain were crossed to $D$. sechellia males and the $F_{1}$ hybrid females were backcrossed to $D$. simulans $\left(f^{2} ; n t p m\right.$; st $e)$ males producing offspring with different combinations of $D$. simulans and $D$. sechellia chromosomes. The identity of these chromosomes or chromosomal regions was read from the individual's phenotype. By examining a trait of each backcross phenotype, one can determine the effect of each chromosomal combination on that trait. We examined four different traits between species or between species and their hybrids: sperm and testis lengths, hybrid male sperm motility, and viability of backcross offsprings. The reciprocal $F_{1}$ (the cross between $D$. sechellia females and $D$. simulans males) was not successful. All stocks and crosses were reared in 8-dram vials on banana medium at $21-23^{\circ} \mathrm{C}$.

\section{Interspecific $Y$ chromosome introgression}

To examine the effect of the $\mathrm{Y}$ chromosome on sperm length between $D$. simulans and $D$. sechellia, we introgressed the $\mathrm{Y}$ chromosome of $D$. sechellia into the background of $D$. simulans. The method used is the same as described in Zeng \& Singh (1993b). $F_{1}$ hybrid females were backcrossed to males of $D$. sechellia and the resulting backcross males were crossed to $D$. simulans females. The males produced in this cross, which carried the $\mathrm{Y}$ chromosome of $D$. sechellia, were then crossed to $D$. simulans females repeatedly to replace other chromosomes by those of $D$. simulans.

\section{Testis dissection, slide preparation and sperm length measurement}

Broken sperm are indistinguishable from intact sperm, so it is problematic to measure individual sperm longer than $1 \mathrm{~mm}$ directly. Instead, the length of bundles of fully elongated mature cysts were measured in the testis. Sperm and mature cysts of one species have similar lengths and the latter could be identified as a fully elongated cyst by the characteristic aspects of the extremities (one is occupied by the sperm head which begins to spread and the other by what will form the waste bag at the sperm individualization stage). This method allows rapid measurement of large numbers of samples. Purespecies males and backcross males with different phenotypes were dissected in saline solution (Ringer) on microscope slides using a pair of forceps and a dissecting needle. Testes were separated from seminal vesicles and the rest of the male reproductive tract. Cysts were spread out of the testes, and separated from each other by using a very fine needle. The slides with the cysts or the testis (only one testis per male) were allowed to dry at room temperature, fixed with 95 per cent ethanol for 4 min, dried again, and then stained with 2 per cent aceto-orcein solution. They were finally washed with distilled water, dried and sealed with permanent mounting medium.

The cyst and testis length measurements were aided by using the public domain program $\mathrm{N} 1 \mathrm{H}$ IMAGE. This program (written by W. Rasband at the US National Institutes of Health) can be obtained through anonymous ftp from zippy.nimh.nih.gov and runs on a Macintosh computer which was connected 
to a dissecting microscope through a video camera (Hitachi, model VK-C150). Because previous studies have shown little interindividual variation (Joly, 1987), 50 cysts for one male from each parental species and $F_{1}$ hybrids were measured. In the backcross progeny, 15 cysts per male and 25 males per phenotype were analysed. In the following sections, the measures are called sperm length but are made on the cyst. The mean testis length was based on the mean of 25 independent testes for each phenotype.

\section{Results}

$\mathrm{F}_{1}$ male hybrids between $D$. simulans females and $D$. sechellia are perfectly viable but sterile. To investigate the autosomal or sex chromosomal contribution to male viability and sterility we have analysed the backcross progeny from the $F_{1}$ females $(D$. simulans $\times D$. sechellia) crossed to $D$. simulans males. The viability, sperm motility, sperm length and testis size were estimated for each phenotypic class and were compared to parental classes to investigate the genetic effects on these characters.

Table 1 Number of males, observed and expected, from 16 backcross phenotypic classes having the $\mathrm{X}$ chromosome from Drosophila simulans, and two backcross phenotypic classes with nonrecombinant autosomes which carry the D. sechellia $\mathrm{X}$ chromosome

\begin{tabular}{lcc}
\hline & \multicolumn{2}{c}{ Number of males } \\
\cline { 2 - 3 } Phenotypes & Observed & Expected \\
\hline$f^{2} ; n t p m ;$ st $e$ & 626 & 491 \\
$f^{2} ;++;++$ & 608 & 491 \\
$f^{2} ; n t p m ;++$ & 620 & 491 \\
$f^{2} ;++; s t e$ & 550 & 491 \\
$f^{2} ; n t+;++$ & 532 & 491 \\
$f^{2} ;+p m ; s t e$ & 530 & 491 \\
$f^{2} ; n t+;$ st $e$ & 423 & 491 \\
$f^{2} ;+p m ;++$ & 677 & 491 \\
$f^{2} ; n t p m ; s t+$ & 127 & 181 \\
$f^{2} ;++;+e$ & 141 & 181 \\
$f^{2} ; n t p m ;+e$ & 121 & 181 \\
$f^{2} ;++; s t+$ & 178 & 181 \\
$f^{2} ; n t+; s t+$ & 62 & 181 \\
$f^{2} ;+p m ;+e$ & 63 & 181 \\
$f^{2} ; n t+;+e$ & 39 & \\
$f^{2} ;+p m ; s t+$ & 80 & \\
Total & 5377 & \\
$+; n t p m ; s t e$ & 440 & \\
$+;++;++$ & 617 & \\
\hline
\end{tabular}

\section{Viability effects}

The $\mathrm{X}$ chromosomal effect on hybrid viability can be determined by comparing the same autosomal genotypes segregating with the $D$. simulans or $D$. sechellia $\mathrm{X}$ chromosome. In this experiment the $\mathrm{Y}$ chromosome comes from $D$. simulans. In Table 1 we can see that the $\mathrm{X}$ chromosomes, regardless of their origin (from $D$. sechellia or $D$. simulans), have no effect when autosomes were heterozygous $(1 / 2 \mathrm{D}$. sechellia and $1 / 2 D$. simulans, 617 and 608 , respectively). On the contrary, when autosomes were homozygous from $D$. simulans ( $n t$ pm; st e) the $\mathrm{X}$ chromosome of $D$. sechellia had a significant reduction in the number of progeny (440) compared to the $\mathrm{X}$ chromosome of $D$. simulans progeny (626).

The viability of males from the 16 backcross phenotypes having the $\mathrm{X}$ chromosome from $D$. simulans were analysed as a function of the marker segregation. The expected frequencies of backcross phenotypic classes can be calculated from the levels of recombination, which are a function of the markers' location on the chromosome (given in the Material and methods section). The expected levels of recombination between the markers used in the present study are 0.25 for chromosome 2 , and 0.135 for chromosome 3 . Thus the expected value of the backcross phenotype having the parental chromosome 3 and parental (or recombinant in our case) chromosome 2 is given by the total number of offspring observed (5377) multiplied by the expected frequencies of these chromosomes, which are 0.365 $(0.5-0.135)$ and 0.25 , respectively, $\quad(=5377 \times$ $0.365 \times 0.25=491)$. Similarly, the expected value of the backcross phenotype having recombinant chromosome 3 and parental (or recombinant) chromosome 2 would be: $5377 \times 0.135 \times 0.25=181$. The $\chi^{2}$ value between the observed and expected numbers of males of each phenotype was statistically significant $\left(\chi_{15}^{2}=563.20, P<0.001\right)$. Results show that there is a significant excess of progenies when chromosome 3 is nonrecombinant between $s t$ and $e$, whereas there is a significant deficiency when it is recombinant (Table 1). It should be noted that the deficiency is notably enhanced when both chromosomes 2 and 3 are recombinant.

\section{Sperm motility}

Pure species males, and $F_{1}$ and $F_{2}$ hybrid males with different genotypes were dissected to study sperm motility. The frequencies of males with motile sperm in $D$. simulans and $D$. sechellia are 98 per cent and 95 per cent, respectively (Table 2), which is similar 
Table 2 Measurements of motile sperm and testis and sperm lengths in the backcross males

\begin{tabular}{|c|c|c|c|}
\hline Phenotypes & $\begin{array}{l}\text { Frequency of males } \\
\text { with motile sperm* }\end{array}$ & $\begin{array}{l}\text { Sperm length }(\mathrm{mm}) \\
\text { mean } \pm \operatorname{SE}(n=25)\end{array}$ & $\begin{array}{l}\text { Testis length }(\mathrm{mm}) \\
\text { mean } \pm \mathrm{SE}(n=25)\end{array}$ \\
\hline simulans & $0.98(100)$ & $1.161 \pm 0.006$ & $1.264 \pm 0.022$ \\
\hline sechellia & $0.95(100)$ & $1.598 \pm 0.005$ & $1.683 \pm 0.016$ \\
\hline $\mathrm{F}_{1}$ (sise) & $0.55(154)$ & $1.138 \pm 0.012$ & $1.491 \pm 0.016$ \\
\hline $\mathbf{1} f^{2} ;$ nt pm; st e & $0.73(157)$ & $1.222 \pm 0.006$ & $1.423 \pm 0.014$ \\
\hline $2+; n t p m ; s t e$ & $0.03(194)$ & $1.177 \pm 0.026$ & $0.947 \pm 0.047$ \\
\hline $\mathbf{3} f^{2} ;+p m ;$ st $e$ & $0.53(134)$ & $1.212 \pm 0.008$ & $1.402 \pm 0.032$ \\
\hline $4 f^{2} ; n t+; s t e$ & $0.60(120)$ & $1.185 \pm 0.014$ & $1.422 \pm 0.021$ \\
\hline $5 f^{2} ; n t p m ;+e$ & $0.43(65)$ & $1.172 \pm 0.018$ & $1.546 \pm 0.029$ \\
\hline $6 f^{2} ;$ nt pm; st + & $0.60(78)$ & $1.225 \pm 0.010$ & $1.558 \pm 0.023$ \\
\hline $7 f^{2} ;$ nt pm; ++ & $0.55(131)$ & $1.242 \pm 0.008$ & $1.493 \pm 0.019$ \\
\hline $8 f^{2} ;++;$ st $e$ & $0.56(115)$ & $1.193 \pm 0.007$ & $1.465 \pm 0.021$ \\
\hline $9 f^{2} ; n t+;++$ & $0.45(110)$ & $1.213 \pm 0.012$ & $1.489 \pm 0.016$ \\
\hline $10 f^{2} ;+p m ;++$ & $0.51(117)$ & $1.208 \pm 0.011$ & $1.551 \pm 0.023$ \\
\hline $11 f^{2} ;++; s t+$ & $0.48(116)$ & $1.201 \pm 0.007$ & $1.676 \pm 0.019$ \\
\hline $12 f^{2} ;++;+e$ & $0.57(100)$ & $1.151 \pm 0.012$ & $1.650 \pm 0.022$ \\
\hline $13 f^{2} ;++;++$ & $0.40(116)$ & $1.205 \pm 0.010$ & $1.544 \pm 0.017$ \\
\hline $14+;++;++$ & $0.06(363)$ & $1.180 \pm 0.010$ & $1.510 \pm 0.040$ \\
\hline
\end{tabular}

*The number of males dissected is given in brackets. Fifteen cysts were measured for each male. $n=$ number of males analysed; sise $=$ Drosophila simulans female crossed to D. sechellia males; the backcross phenotypes are numbered for clearer explanation in the text.

to results obtained by Coyne \& Kreitman (1986). About half of the $\mathrm{F}_{1}$ hybrid males $(D$. simulans females $\times D$. sechellia males) have some motile sperm in their seminal vesicles; this is almost more than 10 times the value obtained by these previous authors. However, these males are also sterile when tested by sib matings. In the backcross generation, the proportions of males with motile sperm vary among different genotypes and are similar to the results obtained by Coyne \& Kreitman (1986). Consistent with the findings of these authors, a dramatic effect of the $\mathrm{X}$ chromosome on sperm motility is observed when the marked region of the $\mathrm{X}$ chromosome is from $D$. sechellia (the percentages of sperm motility are 3 per cent and 6 per cent, $\left.\chi_{1}^{2}=102.55, P<0.001\right)$. When the $\mathrm{X}$ chromosome region marked by forked is from $D$. simulans, the proportions range from 40 to 73 per cent. A test for homogeneity on the percentages of backcross males having motile sperm is statistically significant $\left(\chi_{11}^{2}=43.98, P<0.001\right.$, Table 3$)$. From this global test, it is possible to decompose the effects of various autosomes by performing a partition of $\chi^{2}$ tests (Lancaster, 1950). The most important contribution (67 per cent) to this heterogeneity corresponds to the nonrecombinant autosomal genotypes $\left(f^{2} ; n t p m\right.$, st $e$ and $\left.f^{2} ;++;++\right)$ indicating a strong major effect of the entire species-specific autosomal composition. The comparison of nonrecombinant chromosome 2 with chromosome 3 gave a statistically significant difference, accounting for 11.8 per cent of the total variability $\left(\chi_{1}^{2}=5.19, P<0.05\right)$. All the other effects are not statistically significant.

\section{Sperm length}

Sperm length distributions for $D$. simulans, $D$. sechellia and the $F_{1}$ hybrids are given in Fig. 1 and Table 2. The sperm length means of the two parental species are significantly different (1.161土 $0.006 \mathrm{~mm}$ for $D$. simulans and $1.598 \pm 0.005$ for $\bar{D}$. sechellia, Table $2, t$-test, $P<0.05)$. In sperm length, the $F_{1}$ hybrid males $(D$. simulans females $\times D$. sechellia males) are statistically similar to $D$. simulans males but different from $D$. sechellia ( $t$-test, sequential Bonferroni procedure, $P<0.05$, Weir, 1990). The absence of the reciprocal cross $(D$. sechellia females $\times D$. simulans males) prevents any conclusion on the effects of $\mathrm{X}$ or $\mathrm{Y}$ chromosomes, or even on maternal effect on cyst length. However, the results of the backross from $F_{1}$ female hybrids crossed by $D$. simulans males provide an opportunity to obtain a 
Table $3 \chi^{2}$ analysis for testing the effect of parental and recombinant autosomes on sperm motility. The number in brackets represents the contribution of each $\chi^{2}$ to the total

\begin{tabular}{|c|c|c|c|c|c|}
\hline Phenotypes & & & $\begin{array}{c}\text { Levels of analysis } \\
\chi^{2}=43.98(\text { d.f. }=11)\end{array}$ & & \\
\hline $\left.\begin{array}{l}f^{2} ; \text { nt pm; st e } \\
f^{2} ;++;++\end{array}\right\}$ & $29.8(67.76 \%)^{*}$ & & & & \\
\hline $\left.\begin{array}{l}f^{2} ; n t p m ; s t+ \\
f^{2} ;++;+e\end{array}\right\}$ & $0.19(0.43 \%)$ & $519(1180 \%) *$ & & & \\
\hline $\left.\begin{array}{l}f^{2} ; \text { nt pm; }++ \\
f^{2} ;++; \text { st e }\end{array}\right\}$ & $0.01(0.02 \%) \quad$ & $0.19(11.00 \%)$ & $4.3 \times 10^{-5}(0 \%)$ & & $2.72(6.18 \%)$ \\
\hline $\left.\begin{array}{l}f^{2} ; n t+;++ \\
f^{2} ;+ \text { pm; st e }\end{array}\right\}$ & $1.11(2.52 \%)$ & $2.04(4.64 \%)$ & & $0.64(1.46 \%)$ & \\
\hline $\left.\begin{array}{l}f^{2} ; n t+; s t e \\
f^{2} ;+p m ;+++\end{array}\right\}$ & $1.82(4.14 \%)$ & & & & \\
\hline $\left.\begin{array}{l}f^{2} ; n t p m ;+e \\
f^{2} ;++; s t+\end{array}\right\}$ & $0.45(1.02 \%)$ & & & & \\
\hline
\end{tabular}

*Significant at $P<0.05$.

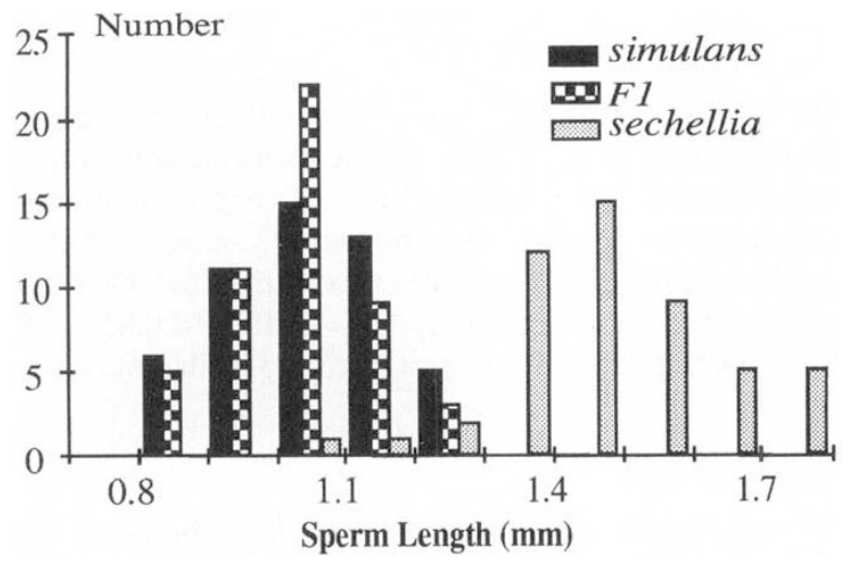

Fig. 1 Sperm length distribution for Drosophila simulans, $D$. sechellia and their $F_{1}$ hybrid $(D$. simulans females $\times D$. sechellia males). Fifty cysts were measured in each case.

genetic constitution with an $\mathrm{X}$ chromosome from $D$. sechellia and a Y chromosome from $D$. simulans, and hence to discriminate between an effect caused by sex chromosomes, autosomes or maternal effect (Table 2). The comparison of phenotypes 1 and 2 on the one hand, and 13 and 14 on the other, gave no statistically significant results $(t$-tests, $P>0.05)$ indicating that the $\mathrm{X}$ chromosome has no effect on the cyst length, whether the autosomal constitution is homozygous $D$. simulans or heterozygous, nor any maternal factor. With these results, it is possible to conclude that the similarity of the sperm lengths obtained in $F_{1}$ hybrids and in pure $D$. simulans species clearly indicates an autosomal dominant effect.

\section{Testis length}

Previous studies have shown a strong correlation between sperm and testis length in the Drosophilidae family (Joly \& Bressac, 1994). This seems to indicate a functional relationship. One could raise the question of a possible genetic relationship between these two traits. Mean testis lengths are significantly different between $D$. simulans, $D$. sechellia and the $F_{1}$ hybrids in all pairwise comparisons ( $t$-test, sequential Bonferroni procedure, $P<0.05$, Table 2). In this context the analysis of testis length could represent one of the better ways to further the genetic analysis of hybrid sterility. The measurements were performed on 14 backcross phenotypes (12 having greater numbers of individuals with the $\mathrm{X}$ from $D$. simulans which excluded the four double recombinants and two with the $X$ from D. sechellia). This was undertaken so that the effect of each chromosomal arm could be determined in four independent pairwise tests between backcross 
phenotypes $\left(f^{2} ; n t p m ;\right.$ st $e$ and $n t p m ;$ st $e$ to test the effect of chromosome X).

In the backcross progenies one would expect, if the genetic determination is simple, the testis size segregation to be correlated with that of the chromosomal markers. The results show that the testis length in the backross progeny is, in general, higher than that of the $D$. simulans and $F_{1}$ hybrids (Table 2). This indicates a complex genetic determination with perhaps an extrachromosomal effect. The most important result is the drastic reduction of the testis size, which is significantly inferior to all other classes, when the $\mathrm{X}$ chromosome from $D$. sechellia is associated with $D$. simulans autosomes ( $0.947 \mathrm{~mm}$, phenotype 2 , Table 2 ). This strong interaction is only caused by homozygous autosomes because when they are heterozygous (phenotype 14) the testis size is comparable to that of $F_{1}$ hybrids where the $\mathrm{X}$ chromosome is from $D$. simulans.

There is no major effect of the chromosome 2 on the testis size, whether it is recombinant or not, when the chromosome 3 is homozygous $D$. simulans (comparison of the classes 1, 3, 4, 8). On the other hand, the testis size is significantly enhanced when the chromosome 2 is homozygous $D$. simulans with the $D$. sechellia $3 \mathrm{~L}$ (class 5) or 3R (class 6), or greatly enhanced when it is heterozygous with the $D$. sechellia $3 \mathrm{~L}$ (class 11 ) or $3 \mathrm{R}$ (class 12 , Table 4 ). This indicates a significant effect of $D$. sechellia chromosome 3, each chromosomal arm contributing to enhance the testis size. However, the two effects on the $3 \mathrm{~L}$ and $3 \mathrm{R}$ seem to be lost when combined because the testis size is smaller when chromosomes 2 and 3 are heterozygous (class 13), in comparison to that of the individuals having the chromosome 2 heterozygous and the chromosomes $3 \mathrm{~L}$ or $3 \mathrm{R}$ recombinant (classes 11, 12). This result suggests that chromosomes $3 \mathrm{~L}$ and $3 \mathrm{R}$ both possess a major effect which interacts with the $D$. sechellia chromosome 2 (comparison of the classes 5,6 and 11,12 ).

\section{Effect of interspecific $Y$ chromosome introgression on sperm length}

There was a significant difference of the sperm length between the introgressed $D$. sechellia Y chromosome in a $D$. simulans background and the pure $D$. simulans strain ( $t$-test, $P<0.01$, Fig. 2 ) indicating the presence of a factor on the $\mathrm{Y}$ chromosome influencing sperm length. Sperm are shorter with the $D$. sechellia Y chromosome.

\section{Discussion}

Four traits involved in species differences (hybrid inviability, sperm motility, sperm length and testis size) were analysed in order to understand further the genetic basis of speciation. Between-population variability in sperm size or structure, a possible basis for incipient speciation, is poorly documented and so far has been studied mainly in polychaete organisms (Franzén, 1956; Gibbs, 1971). In the Drosophilidae family the sperm length variation has previously been documented as a large interspecific variability of tiny, giant or heteromorphic sperm (Joly et al., 1989) but nothing is known about the genetic bases of these differences.

The reduction in the number of progeny obtained in the backcross experiment, the small percentage of motile sperm (shown previously by Coyne \& Kreit-

Table 4 Autosomal epistatic effects on the testis size of hybrid males from the cross $F_{1}$ females $\times D$. simulans males (in the comparison involving recombinant chromosomes 2 and 3 , the $D$. simulans left arm with $D$. sechellia right arm should be considered against the opposite, $D$. sechellia left arm with $D$. simulans right arm)

\begin{tabular}{lccc}
\hline & \multicolumn{3}{c}{ Maternal chromosome 3 } \\
\cline { 2 - 4 } X. simulans & simulans & Recombinant & sechellia \\
\hline $\begin{array}{l}\text { Maternal chromosome } 2 \\
\text { simulans }\end{array}$ & $1.423 \pm 0.014$ & $1.546 \pm 0.029$ & $1.493 \pm 0.019$ \\
& & $1.558 \pm 0.023$ & \\
Recombinant & $1.402 \pm 0.032$ & - & $1.489 \pm 0.016$ \\
& $1.422 \pm 0.021$ & & $1.551 \pm 0.023$ \\
sechellia & $1.465 \pm 0.021$ & $1.676 \pm 0.019$ & $1.544 \pm 0.017$ \\
& & $1.650 \pm 0.022$ & \\
\hline
\end{tabular}




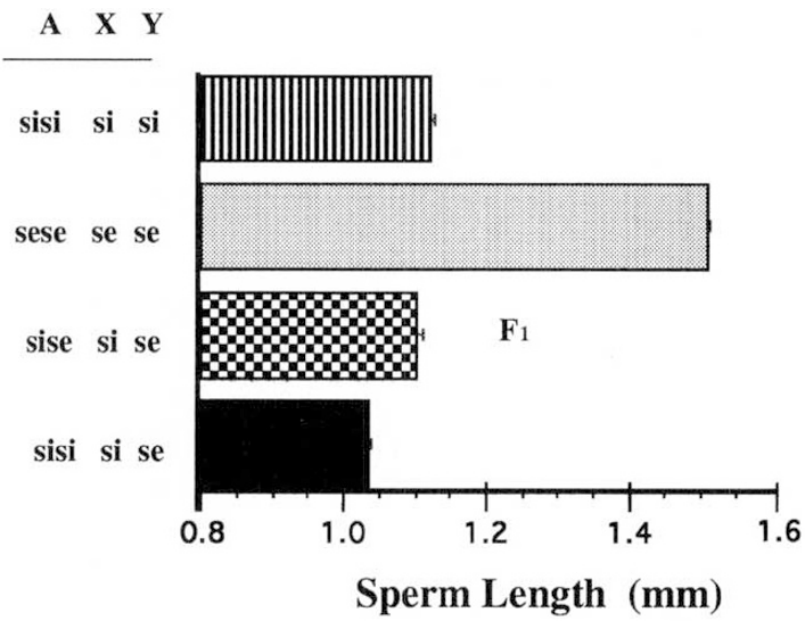

Fig. 2 Effect of Y chromosome on sperm length. The chromosomal constitution of each strain is indicated on the left (se, Drosophila sechellia; $\mathrm{si}, D$. simulans).

$\mathrm{A}=$ autosomes.

man, 1986) and the decrease in testis length, all demonstrate a strong incompatibility between the $D$. sechellia $\mathrm{X}$ chromosome and $D$. simulans autosomes. As first noted by Muller (1942), all hybrid incompatibilities must be asymmetric. This is confirmed here by the fact that the interaction between the $D$. simulans $\mathrm{X}$ chromosome with heterozygous autosomes gives a similar number of offspring to that obtained in control cases. The incompatibility between the $D$. sechellia $\mathrm{X}$ chromosome and $D$. simulans autosomes should be observed in different segregation of autosomes in the backcrosses involving hybrid $F_{1}$ females (from $D$. simulans females $\times D$. sechellia males) and $D$. simulans males, and this should hide the effects caused by autosomes. That is why only the backcross genotypes with a $D$. simulans $\mathrm{X}$ chromosome were studied here. In addition to this strong incompatibility between the sex chromosome and autosomes, an epistatic interaction between chromosomes 2 and 3 was observed for hybrid inviability and sperm motility.

The crosses between these two species show that $D$. simulans has dominant autosomal factors for sperm length with respect to those of $D$. sechellia. As a consequence, the effect of individual chromosomes could not be ascertained. Because it is unlikely that mature sperm can develop normally in an abnormally small testis, and because sperm and testis lengths are strongly correlated (Joly \& Bressac, 1994), the latter trait is a good candidate for evaluating the degree of interspecific hybrid sterility. As with sperm length, the testis lengths of $D$. simulans and $D$. sechellia are significantly different. The $F_{1}$ hybrids show intermediate values suggesting an addi- tive effect of the $D$. simulans and $D$. sechellia genes. This result clearly indicates that the two traits, sperm and testis sizes, are independent and can involve separate sets of gene(s). The analysis of the effect of each chromosome in the backcross progeny shows that the size of the testis has a polygenic control with at least major factors localized on chromosomes X, 3L, 3R. Chromosome 3 has a greater effect on these traits of the fertilization system than chromosome 2 . This effect exists in $D$. sechellia $3 \mathrm{~L}$ and $3 \mathrm{R}$ and acts in interaction with $D$. sechellia chromosome 2 . This does not exclude some extrachromosomal contribution and complex effects underlying interspecific chromosomal interaction. This is in accordance with results obtained by Zengh \& Singh (1993a) showing that most divergent testis proteins among species of the $D$. melanogaster complex are associated with autosomes. Furthermore, an additive contribution of chromosomes 2 and 3 is shown when the testis length of $f^{2}$ is compared to that of $f^{2} ; n t ~ p m$ or $f^{2}$; st e (1.544 vs. 1.493 or 1.465 , respectively).

The failure to obtain an $F_{2}$ generation shows that $F_{1}$ hybrid males (from $D$. simulans females mated with $D$. sechellia males) are sterile, even those having motile sperm in the seminal vesicle (55 per cent, Table 2). As previously suggested by several authors, the presence of motile sperm is not enough to test male fertility. In this case, the reproductive isolation is completed probably by an insufficient amount of sperm or abnormalities during sperm transfer or storage, as is the case between $D$. simulans females and D. mauritiana males (Coyne, 1993). A major effect of a factor localized on the $\mathrm{X}$ chromosome is shown by the drastic reduction of males having motile sperm in both phenotypes with an X from $D$. sechellia. Analyses of reproductive isolation almost always reveal a disproportionately large effect of the $\mathrm{X}$ chromosome on hybrid sterility and inviability (Wu \& Beckenbach, 1983; Charlesworth et al., 1987).

The existence of a factor on the $Y$ chromosome is shown here by an introgression of $D$. sechellia $\mathrm{Y}$ in a D. simulans background (Fig. 2). This genetic constitution results in normal, mature sperm, but smaller than those obtained in backcross progenies. This is in contradiction to the preceding results from backcross offspring and indicates the existence of a factor(s) localized on the $\mathrm{Y}$ chromosome which diminishes the size of the gamete. The effect of the $\mathrm{Y}$ chromosome found here is supported by studies on different Drosophila species (Kennison, 1981; Gatti \& Pimpinelli, 1983; Hennig, 1985). Some of them have shown that sperm length could be 
dependent on the number of $Y$ copies in the genome. In $D$. melanogaster and $D$. hydei males lacking a $\mathrm{Y}$ chromosome (XO) the testis length is about half the size of the controls XY or double in XYY males (Hess \& Meyer, 1963; Hanna et al., 1985). However, these results have been contradicted by subsequent analyses (Gould-Somerot et al., 1974). The present results point out the role of the sperm size in their normal function. There is a classical relationship between sperm kinetics parameters (as the beat frequency or amplitude) and the strength of the propulsion of the gamete in the female reproductive tracts (Serres et al., 1984). One possibility is that these parameters grow with the size of the sperm. This can be illustrated by short and long sperm in dimorphic species of the $D$. obscura group (Bressac et al., 1991). One could speculate that shorter sperm of males with a $D$. sechellia $\mathrm{Y}$ in a $D$. simulans background exhibit lower progression velocity than longer sperm of the pure species. Such a sperm velocity difference could change the issue of sperm competition, and this is what was found in sperm displacement/remating ability experiments (Johnson et al., 1993).

In conclusion, the most dramatic result emerging from the present data set is epistatic interactions between autosomes affecting species differences. This pattern can be seen from all the characters studied here. A simple backcross analysis was able to reveal interesting genetic interactions in different morphological traits that differ between species and which are suspected as being the major targets of sexual selection (Singh \& Zeng, 1995). More studies are needed to push the analysis of sperm and testis length still further. The fact that the testis size for the genotypes coming from the backcross progenies is greater than that of the parental species suggests that some modifier genes are being released from their species-specific genetic control. Species-specific modifiers in sexually selected traits may hold the key for rapid evolution of such traits during speciation.

\section{Acknowledgements}

We wish to thank P. Capy, J.R. David, P.-H. Gouyon and D. Lachaise for helpful criticisms on successive drafts of the manuscript. This work was supported by a NATO grant 37C92FR to D.J.

\section{References}

BRESSAC, C., JOLY, D., DEVAUX, J., SERRES, C., FENEUX, D. AND LACHAISE, D. 1991. Comparative kinetics of short and long sperm in sperm dimorphic Drosophila species. Cell Mot. Cytoskel., 19, 269-274.

(C) The Genetical Society of Great Britain, Heredity, 78, 354-362.
CHARlesworth, B., COYNE, J. A. AND BARTON, N. H 1987. Relative rates of evolution of sex chromosomes and autosomes. Am. Nat., 130, 113-146.

COYNE, J. A 1984 . Genetic basis of male sterility in hybrids between two closely related species of Drosophila. Proc. Natl. Acad. Sci. U.S.A., 81, 4444-4447.

COYNE, J. A 1985 . Genetic studies of three sibling species of Drosophila with relationship to theories of speciation. Genet. Res., 46, 169-192.

COYNE, J. A 1992. Genetics and speciation. Nature, 355, $511-515$

COYNE, J. A 1993. The genetics of an isolating mechanism between two sibling species of Drosophila. Evolution, 47, 778-788.

COYNE, J. A. AND KREITMAN, M. 1986. Evolutionary genetics of two sibling species, Drosophila simulans and Drosophila sechellia. Evolution, 40, 673-691.

DOBZHANSKY, T. 1936. Studies on hybrid sterility. II. Localization of sterility factors in Drosophila pseudoobscura. J. Genet., 34, 135-151.

Dobzhansky, т. 1937. Genetics and the Origin of Species. Columbia University Press, New York.

FRANZÉN, A, 1956. On spermiogenesis, morphology of the spermatozoon, and biology of fertilization among invertebrates. Zool. Bidr. Upps., 31, 355-482.

GATTI, M. AND PIMPINELLI, s. 1983. Cytological and genetic analysis of the $\mathrm{Y}$ chromosome of Drosophila melanogaster. I. Organization of the fertility factors. Chromosoma, 88, 349-373.

GIBBS, P. E 1971. A comparative study of reproductive cycles in four polychaete species belonging to the family Cirratulidae. J. Mar. Biol. Ass. U.K., 51, 745-769.

GOULD-SOMEROT, M., HARDY, R. AND HOLLAND, L. 1974. The $\mathrm{Y}$ chromosome and sperm length in $D$. melanogaster. Exp. Cell. Res., 87, 397-398.

HANNA, P. J., GLÄTZER, K. H., LIEBRICH, W. AND HESS, O. 1985. Genetic factors affecting normal growth of testes in Drosophila hydei. Roux's Arch. Dev. Biol., 194, 131-139.

HENNIG, w. 1985. Y chromosome function and spermatogenesis in Drosophila hydei. Adv. Genet., 23, 179-234.

HESS, O. AND MEYER, G. F. 1963. Chromosomal differentiations of the lampbrush type formed by the $Y$ chromosome in Drosophila hydei and Drosophila neohydei. J. Cell Biol., 16, 527-539.

JOHNSON, N. A., HOLlOCHER, H., NOONBURG, E. AND WU, C.-I. 1993. The effects of interspecific $Y$ chromosome replacements on hybrid sterility within the Drosophila simulans clade. Genetics, 135, 443-453.

JOLY, D. 1987. Between species divergence of cyst length distributions in the Drosophila melanogaster species complex. Jap. J. Genet., 62, 257-263.

JOLY, D. AND BRESSAC, C. 1994. Sperm length in Drosophilidae (Diptera): estimation by testis and receptacle length. Int. J. Insect Morphol. \& Embryol., 23, 85-92.

JOLY, D., CARIOU, M.-L., LACHAISE, D. AND DAVID, J. R. 1989. Variation of sperm length and heteromorphism in Drosophilid species. Génét. Sél. Évol., 21, 283-293. 
Joly, D., BRESSAC, C., DEVAuX, J. AND LACHAISE, D. 1991. Sperm length diversity in Drosophilidae. Drosoph. Inf. Serv., 72, 104-108.

JOLY, D., BRESSAC, C. AND LACHAISE, D. 1995. Disentangling giant sperm. Nature, 377, 202.

KENNiSON, J. A 1981. The genetic and cytological organization of the $Y$ chromosome of Drosophila melanogaster. Genetics, 98, 529-548.

LACHAISE, D., DAVID, J. R., LEMEUNIER, F., TSACAS, L, AND ASHBURNER, M. 1986. The reproductive relationships of Drosophila sechellia with $D$. mauritiana, D. simulans and D. melanogaster from the afrotropical region. Evolution, 40, 262-271.

LANCASTER, H. O 1950. The exact partition of $\chi^{2}$ and its application to the problem of pooling of small expectations. Biometrika, 37, 267-270.

LEMEUNIER, F. AND ASHBURNER, M. 1984. Relationships within the melanogaster species subgroup of the genus Drosophila (Sophophora). IV. The chromosomes of two new species. Chromosoma, 89, 343-351.

MULLER, H. J 1942. Isolating mechanisms, evolution and temperature. Biol. Symp., 6, 71-125.

ORR, H. A 1987 . Genetics of male and female sterility in hybrids of Drosophila pseudoobscura and $D$. persimilis. Genetics, 116, 555-563.

ORR, H. A 1989. Genetics of sterility in hybrids between two subspecies of Drosophila. Evolution, 43, 180-189.

ORR, H. A 1991. Is single-gene speciation possible? Evolution, 45, 764-769.

PANTAZIDIS, A. C., Galanopoulos, V. K. AND zouros, E. 1993. An autosomal factor from Drosophila arizonae restores normal spermatogenesis in Drosophila mojavensis males carrying the $D$. arizonae $Y$ chromosome. Genetics, 134, 309-318.
SERRES, C., FENEUX, D., JOUANNET, P. AND DAVID, G. 1984. Influence of the flagellar wave development and propagation on the human sperm movement in seminal plasma. Gamete Res., 9, 183-195.

SINGH, R. S. AND ZENG, L. W 1995. Genetic divergence, reproductive isolation and speciation. In: Golding, B. (ed.) Non-neutral Evolution. Theories and Molecular Data, pp. 217-232. Chapman and Hall, New York.

weIr, B. s 1990. Genetic Data Analysis. Sinauer Associates, Sunderland, MA.

WU, C. I. AND BECKENBACH, A. T 1983. Evidence for extensive genetic differentiation between the sex-ratio and the standard arrangement of Drosophila pseudoobscura and $D$. persimilis and identification of hybrid sterility factors. Genetics, 105, 71-86.

ZENG, L.-W. AND SINGH, R. S 1993a. A combined classical genetic and high resolution two-dimensional electrophoretic approach to the assessment of the number of genes affecting hybrid male sterility in Drosophila sechellia. Genetics, 135, 135-147.

ZENG, L.-W. AND SINGH, R. S 1993b. The genetic basis of Haldane's rule and the nature of asymmetric hybrid male sterility among Drosophila simulans, Drosophila mauritiana and Drosophila sechellia. Genetics, 134, $251-260$.

ZENG, L.-W. AND SINGH, R. S 1995. A general method for identifying major hybrid male sterility genes in Drosophila. Heredity, 75, 331-341.

zouros, E. 1981. An autosome- $Y$ chromosome combination that causes sterility in $D$. mojavensis $\times D$. arizonae hybrids. Drosoph. Inf. Serv., 56, 167-168.

zouros, E., LOFDAHL, K. AND MARTIN, P. 1988. Male hybrid sterility in Drosophila: interactions between autosomes and sex chromosomes in crosses of $D$. mojavensis and D. arizonensis. Evolution, 42, 1332-1341. 